\section{Use of sonoelastography to evaluate texture modifications of Mozzarella di Bufala Campana Protected Designation of Origin during storage at different temperatures}

\author{
Nicola Costanzo, ${ }^{1}$ \\ Adriano Michele Luigi Santoro, ${ }^{2}$ \\ Eleonora Sarno, ${ }^{2}$ Antonio Di Loria, ${ }^{1}$ \\ Rosa Daniela Grembiale, ${ }^{3}$ \\ Domenico Britti, ${ }^{1}$ Federico Capuano ${ }^{4}$ \\ 'Department of Life Sciences, University \\ of Catanzaro Magna Græcia, Catanzaro; \\ 2Department of Veterinary Medicine and \\ Animal Productions, University of Naples \\ Federico II, Naples; ${ }^{3}$ Department of \\ Medical and Surgical Sciences, University \\ of Catanzaro Magna Græcia, Catanzaro; \\ ${ }^{4}$ Laboratory of Applied Biotechnologies, \\ Department of Food Inspection, Institute \\ for Experimental Veterinary Medicine of \\ Southern Italy, Portici, (NA), Italy
}

\begin{abstract}
Mozzarella cheese from buffalo milk is a fresh, stringy-textured dairy product, exhibiting a porcelain white colour, a smooth, bright, and humid surface, an extremely thin rind and delicate taste. The high humidity typical of this cheese reduces its shelf-life and it is cause of dramatic organoleptic changes during storage. In this study we tested sonoelastography to evaluate texture changes of mozzarella cheese from buffalo milk during storage. Cheeses form local market produced in the same condition were divided in three batches and stored in different conditions: the first (B1) was stored in preserving liquid at room temperature $\left(20^{\circ} \mathrm{C}\right)$; the second $(\mathrm{B} 2)$ was stored without preserving liquid at $4^{\circ} \mathrm{C}$; and the third (B3) was stored at $4^{\circ} \mathrm{C}$ in preserving liquid. In $\mathrm{B} 1$ sonoelastography showed a reduction of the hardness and stiffness of rind, while in B2 inelastic tissue increased its thickness. Best results were obtained in B3, where no significant difference was evidenced during storage.
\end{abstract}

\section{Introduzione}

L'utilizzo degli ultrasuoni in campo alimentare si è molto diffuso nel corso degli anni grazie alla capacità di fornire informazioni sulle proprietà acustiche degli alimenti e sulla loro capacità di correlarsi ai parametri di qualità (Povey et al., 1988). L'ultrasonografia si dimostra una tecnica non invasiva, di rapida esecuzione e in grado di fornire un'analisi digitale in real-time. Tra le metodiche di studio ecografico, una recente evoluzione è rappresentata dall'elastosonografia, una tecnologia in grado di caratterizzare le risposte elastiche dei tessuti quando sottoposti alle applicazioni di forze esterne deformanti. L'applicazione, mediante una sonda ecografica, di una pressione bilanciata e perpendicolare sull'area oggetto di studio, fa si che gli apparecchi ecografici siano in grado, attraverso algoritmi dedicati, di comparare le variazioni di propagazione delle onde ultrasonore nel tessuto, prima e dopo la compressione. La compressione dei tessuti determina, infatti, una modificazione del segnale trasmesso rispetto a quello ricevuto generando cosi immagini per le quali è possibile ottenere l'analisi ed il calcolo qualitativo dei coefficienti di elasticità. Considerando che la compattezza di un tessuto è direttamente proporzionale alla capacità di condurre più velocemente le onde ultrasonografiche, l'analisi elastosonografica sarà in grado di generare in funzione della loro deformazione un'immagine caratterizzata da diversi livelli cromatici corrispondenti a differenti coefficienti elastici del tessuto. Sviluppata in campo medico umano, l'elastosonografia è stata utilizzata soprattutto nella diagnosi di tumori mammari (Tanter et al., 2008; Berg et al., 2012), per la stadiazione della fibrosi epatica (Bavu et al., 2011) e per lo studio della elasticità dei muscoli (Gennisson $e t$ al., 2010). In campo alimentare, la sua applicazione è stata rivolta allo studio dei cambiamenti strutturali che interessano il latte durante il processo di coagulazione. Tali studi sono stati condotti sia su soluzioni di caseina (Wang et al., 2007), che su latte ricostituito (Ay e Gunasekaran, 1994; Benguigui et al., 1994; Bakkali et al., 2001; Nassar et al., 2001, 2004) nonché su latte intero (Koc e Ozer, 2008). Scopo del presente lavoro è di valutare l'impiego dell'elastosonografia nella caratterizzazione tissutale della Mozzarella di Bufala Campana denominazione di origine protetta (D.0.P.) in funzione di diverse condizioni di conservazione.

\section{Materiali e Metodi}

La Mozzarella di Bufala Campana D.0.P. prodotta nelle stesse condizioni e appartenente allo stesso lotto di produzione, è stata prelevata in un caseificio della provincia di Caserta. Il formaggio è stato diviso in tre gruppi che sono poi stati stoccati in condizioni diverse per 5 giorni successivi al campionamento: il primo (B1) è stato conservato in liquido di governo a temperatura ambiente $\left(+20^{\circ} \mathrm{C}\right)$, il secondo
Correspondence: Nicola Costanzo, Department of Life Sciences, University of Catanzaro Magna Græcia, viale Europa, Edificio delle Bioscienze 88100, Catanzaro (CZ), Italy.

Tel. +39.0961.3694203.

E-mail: costanzo.nic@unicz.it

Key words: Mozzarella di Bufala Campana P.D.O.; Sonoelastography; Texture modifications; Temperature.

Conflict of interest: the authors declare no potential conflict of interest.

Received for publication: 9 October 2014.

Revision received: 29 December 2014.

Accepted for publication: 8 January 2015.

This work is licensed under a Creative Commons Attribution-NonCommercial 3.0 International License (CC BY-NC 3.0).

@C Copyright N. Costanzo et al., 2015

Licensee PAGEPress, Italy

Italian Journal of Food Safety 2015; 4:4746

doi:10.4081/ijfs.2015.4746

(B2) è stato conservato senza liquido di governo a $4^{\circ} \mathrm{C}$ e il terzo (B3) stoccato alla stessa temperatura del secondo ma con liquido di governo. I gruppi sono stati sottoposti ad indagine elastosonografica il giorno stesso (T0), dopo 1 giorno (T1), dopo 2 giorni (T2) e dopo 5 giorni (T3) dal campionamento.

Il principio di base dell'elastosonografia risiede nel fatto che la compressione del tessuto esaminato con la sonda dell'ecografo produce una distorsione, minore nei tessuti duri e maggiore nei tessuti soffici, che può essere rilevata $\mathrm{e}$ quantificata attraverso una rielaborazione di dati effettuata dall'ecografo. Per l'indagine elastosonografica è stato impiegato un ecografo MYLAB 50 XVISION (Esaote, Indianapolis, IN, USA), fornito di sonda ad altra frequenza Elaxto (Esaote). L'elasticità è stata valutata in due aree sferiche di 11,64 mm² (Z1 e Z2), a una profondità dal punto di contatto della sonda rispettivamente di Z1: 0,5 cm e Z2: 1,5 cm. La scelta delle aree e la profondità delle stesse derivano dal tipo di sonda utilizzato: l'aumento della frequenza porta, infatti, ad una ridotta capacità di penetrazione del fascio ultrasonoro. L'applicazione del software dedicato ha permesso di elaborare la propagazione delle onde di compressione, restituendo così oltre alla classica immagine B-mode, l'indice di elasticità relativa (El) all'area selezionata, il rapporto tra le due aree oggetto di campionamento e l'immagine in scala di colori così codificata: rosso (morbido), verde (grado intermedio di rigidità) e blu (duro, anelastico). Una volta appoggiata, la sonda produce una compressione che genera un'onda elastica la cui velocità di trasmissione è registrata dall'apparecchio ed è direttamente proporzionale alla rigidità del tessuto. La forza di compressione viene misurata in tempo reale dal 
software in dotazione all'ecografo mediante una misurazione basata su una scala di 6 punti. A tal riguardo ogni acquisizione è stata ottenuta a un valore di 5 della scala ovvero al limite minimo stabilito dall'ecografo per considerare attendibili le successive misurazioni. Inoltre al fine di valutare l'evoluzione dell'elasticità nel corso dello stoccaggio, l'area di applicazione della sonda ecografica è stata delimitata da uno stampo e impiegata per tutte le acquisizioni di immagini nei tempi T0, T1, T2 e T3. Per ogni prova sono state acquisite immagini multiple e l'elastogramma con la migliore definizione d'immagine è stato utilizzato per la valutazione sulla base della scala dei colori. I dati ottenuti sono stati elaborati con l'analisi della varianza mediante l'utilizzo del software GraphPad Prism (versione 5.00 per Windows; GraphPad Software, San Diego, CA, USA).

\section{Risultati}

I risultati dell'indagine elastosonografica sono riportati nella Figura 1. I valori di El medi hanno oscillato in B1 da un minimo di 18 ad un massimo di 56, in B2 da un minimo di 25 ad un massimo di 53 mentre in B3 da un minimo di 25 ad un massimo di 57. I risultati degli El sono riportati nella Tabella 1 . Il rapporto di elasticità (Z2/Z1) ha oscillato per B1 da un minimo di 1,06 ad un massimo di 1,8, per B2 da un minimo di 0,93 ad un massimo di 1,71 mentre per B3 da un minimo di 1,46 ad un massimo di 1,82 . Differenze significative $(\mathrm{P}<0.05)$ sono state evidenziate tra i valori di elasticità media in B2 tra Z1 e Z2, mentre comparando i dati delle due aree di campionamento negli altri due sistemi di stoccaggio nessuna differenza significativa è stata evidenziata.

\section{Discussione}

Il lavoro riporta i primi dati riguardanti l'utilizzo della tecnica elastosonografica applicata alla Mozzarella di Bufala Campana D.0.P. I risultati ottenuti dimostrano che l'elastosonografia è sensibile alle variazioni che si verificano durante lo stoccaggio del prodotto in con- dizioni diverse di conservazione. I valori di elasticità media in T0 sono diversi nei singoli lotti, tale differenza va probabilmente imputata all'origine artigianale del prodotto che prevede la filatura a mano e quindi una sostanziale differenza di tessitura. Inoltre l'assenza di significatività statistica tra i diversi metodi di stoccaggio va ascritta alla limitata area di valutazione dell'elasticità. Dalle immagini acquisi-

Tabella 1. Variazione dell'elasticità nel corso dello stoccaggio.

\begin{tabular}{|c|c|c|c|c|}
\hline Tempo & Parametri & & trupp & \\
\hline & & B1 & B2 & B3 \\
\hline T0 & Rapporto elx2/1 & 1,35 & 0,93 & 1,46 \\
\hline & Ell medio & 30 & 43 & 31 \\
\hline & Ell ds & 12,5 & 9,2 & 12,4 \\
\hline & El2 medio & 46 & 40 & 51 \\
\hline & El2 ds & 16 & 12 & 9,3 \\
\hline $\mathrm{T} 1$ & Rapporto elx2/1 & 1,8 & 1,2 & 1,65 \\
\hline & Ell medio & 25 & 34 & 27 \\
\hline & Ell ds & 6,3 & 17,9 & 10,9 \\
\hline & El2 medio & 56 & 43 & 54 \\
\hline & El2 ds & 16 & 8,4 & 7,5 \\
\hline $\mathrm{T} 2$ & Rapporto elx2/1 & 1,54 & 1,64 & 1,82 \\
\hline & Ell medio & 29 & 27 & 25 \\
\hline & Ell ds & 15,6 & 20,6 & 15,4 \\
\hline & El2 medio & 52 & 53 & 57 \\
\hline & El2 ds & 10,1 & 10,7 & 8,4 \\
\hline T3 & Rapporto elx2/1 & 1,06 & 1,71 & 1,63 \\
\hline & Ell medio & 18 & 25 & 27 \\
\hline & Ell ds & 9,8 & 19,3 & 17,1 \\
\hline & El2 medio & 41 & 53 & 53 \\
\hline & El2 ds & 6,3 & 6,2 & 6,5 \\
\hline
\end{tabular}

$\mathrm{B} 1$, gruppo conservato in liquido di governo a temperatura ambiente $\left(+20^{\circ} \mathrm{C}\right)$; $\mathrm{B} 2$, gruppo conservato senza liquido di governo a $4^{\circ} \mathrm{C}$; B3, gruppo stoccato alla stessa temperatura del secondo ma con liquido di governo; $\mathrm{T} 0$, indagine elastosonografica eseguita il giorno del campionamento; T1, indagine elastosonografica eseguita dopo 1 giorno; T2, indagine elastosonografica eseguita dopo 2 giorni; T3, indagine elastosonografica eseguita dopo 5 giorni; rapporto elx2/1, rapporto del valore di elasticità tra l'area Z2 e l'area Z1; Ell medio, valore di elasticità media nell'area Z1; El1 ds, deviazione standard del valore dell'elasticità nell'area Z1; El2 medio, valore di elasticità media nell'area Z2; El2 ds, deviazione standard del valore dell'elasticità nell'area Z2.
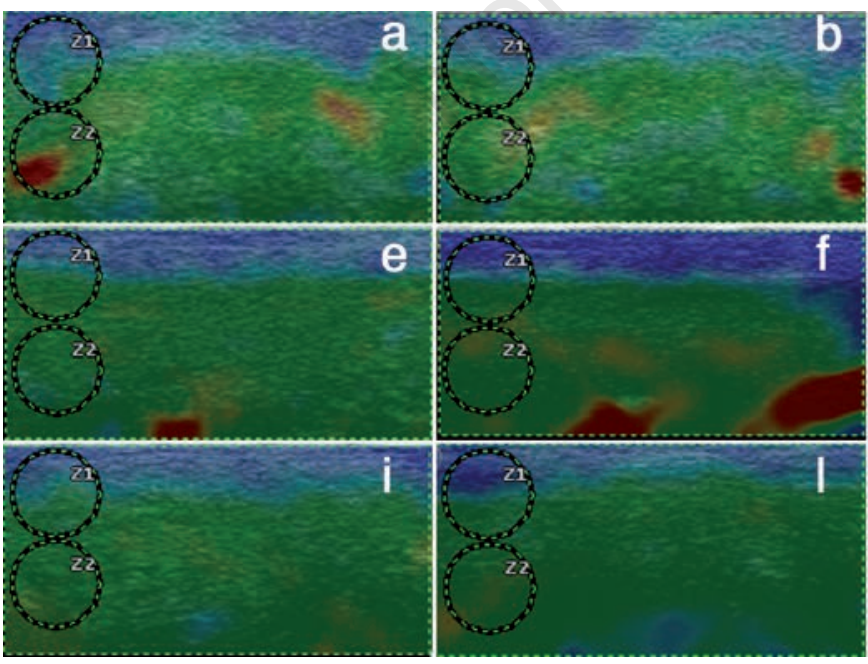
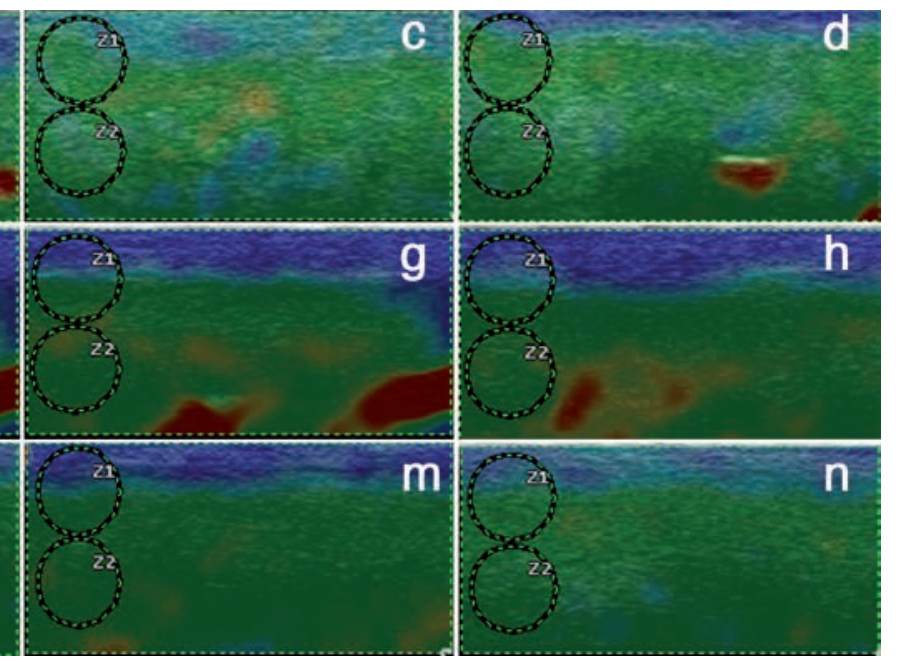

Figura 1. Risultati dell'indagine elastosonografica. a-d) $\mathrm{B} 1$, gruppo conservato in liquido di governo a temperatura ambiente $\left(+20^{\circ} \mathrm{C}\right)$; e-h) B2, gruppo conservato senza liquido di governo a $4^{\circ} \mathrm{C}$; i-n) $\mathrm{B} 3$, gruppo stoccato alla stessa temperatura del secondo ma con liquido di governo. 
te emerge come in $\mathrm{n} \mathrm{B1}$, la conservazione a temperatura ambiente, abbia determinato una diminuzione dell'area blu anelastica corrispondente alla crosta sottile e agli strati sottostanti (Figura 1a-d). In B2 la conservazione a $4^{\circ} \mathrm{C}$ senza liquido di governo ha invece causato, come prevedibile, una disidratazione superficiale del prodotto che si è estesa agli strati sottostanti, determinando un aumento dello spessore blu anelastico, nonché la pressoché, totale scomparsa, delle aree rosse (morbido) presenti in profondità (Figura 1e-h). In B3, il mantenimento del liquido di governo e la conservazione $\mathrm{a}+4^{\circ} \mathrm{C}$ ha consentito invece di preservare le caratteristiche iniziali del prodotto: lo spessore dell'area blu dell'immagine elastosonografica è risultata stabile nel corso dello stoccaggio così come i valori di elasticità risultano stabili sia in Z1 che in Z2 (Figura 1i-n). Lo stoccaggio del prodotto a temperatura di refrigerazione se da un lato è in grado di controllare lo sviluppo microbico (Dellaglio et al., 1993; Coppola et al. 1994, 1995), dall'altro può seriamente influenzare la struttura e la consistenza a seguito di una conservazione prolungata (De Franciscis et al., 1992). L'indagine elastosonografica ha permesso di valutare che non esistono variazioni di elasticità del prodotto quando la conservazione del prodotto a $+4^{\circ} \mathrm{C}$ non supera i 5 giorni successivi alla produzione.

\section{Conclusioni}

L'impiego dell'elastrosonografia si è dimostrato utile nella determinazione dei cambiamenti di elasticità che avvengono nella mozzarella di bufala campana D.0.P. durante lo stoccaggio in diverse condizioni di conservazione. Ulteriori approfondimenti sono però necessari per meglio quantificare le variazioni dell'elasticità del prodotto e per rendere meno empirica la sua valutazione. Tale tecnica ha però il vantaggio di essere facilmente applicabile e ripetibile e di non avere costi aggiuntivi oltre a quelli iniziali nell'acquisto dell'apparecchiatura. È inoltre possibile ipotizzare un suo efficace utilizzo nella filiera della mozzarella di bufala camapana D.0.P. sia per meglio caratterizzare il prodotto e garantirne la conformità a quanto stabilito dal disciplinare sia in corso di produzione, al fine di monitorare la precoce comparsa di difetti quali soft rind (Kindstedt, 1994). Un ulteriore impiego potrebbe essere rappresentato dalla possibilità di applicare tale tecnologia allo studio delle variazioni di elasticità della cagliata al momento della filatura, in modo da fornire al casaro utili informazioni da impiegare nei processi produttivi.

\section{Bibliografia}

Ay C, Gunasekaran S, 1994. Ultrasonic attenuation measurements for estimating milk coagulation time. Trans ASAE 37:85762.

Bakkali F, Moudden A, Faiz B, Amghar A, Maze G, Montero de Espinosa F, Akhnak M, 2001. Ultrasonic measurement of milk coagulation time. Meat Sci Technol 12:2154-9.

Bavu E, Gennisson JL, Couade M, Bercoff J, Mallet V, Badel A, Fink M, Nalpas B, Tanter M, Pol S, 2011. Noninvasive in vivo liver fibrosis evaluation using supersonic shear imaging: a clinical study on 113 hepatitis $\mathrm{C}$ virus patients. Ultrasound Med Biol 37:1365-73.

Benguigui L, Emery J, Durand D, Bunsel JP, 1994. Ultrasonic study of milk clotting. Lait 74:197-206.

Berg WA, Cosgrove DO, Doré CJ, 2012. Shearwave elastography improves the specificity of breast US: the BE1 multinational study of 939 masses. Radiology 262:435-49.

Coppola R, Cinquanta L, Sorrentino E, 1994. Influenza delle modalità di confezionamento sulla qualità di formaggi freschi a pasta filata. In: Atti del Convegno Internazionale Latte e derivati: tra tecnologie e nutrizione; attualità della ricerca, 7 8 giugno 1994, Roma, Italy.

Coppola R, Grazia L, Rossi F, Salzano G, 1995. Influenza delle tecniche di produzione sulla conservabilità di formaggi freschi a pasta filata prodotti con latte pastorizzato. Industrie Alimentari 34:111.
De Franciscis G, Citro G, Correale E, 1992. Tornando alle origini: difficoltà di fabbricazione della mozzarella di bufala. Il latte 17:550.

Dellaglio F, Coppola R, Sorrentinmo E, Rossi F, Salzano G, Sorlini C, 1993. Effect of storage condition on shelf life of Mozzarella cheese. In: Proc. of the 15th International Symposium Novel Approaches towards Food Safety Assurance, 31 August-3 September 1993, Binger/Rhine, Germany.

Gennisson JL, Deffieux T, Macé E, Montaldo G, Fink M, Tanter M, 2010. Viscoelastic and anisotropic mechanical properties of in vivo muscle tissue assessed by supersonic shear imaging. Ultrasound Med Biol 36:789-801.

Kindstedt PS, 1994. Factors that influence the shredding characteristics of Mozzarella cheese. In: Proc. 3rd California Cheese Symposium, University of Davis, CA, USA.

Koc A, Ozer B, 2008. Nondestructive monitoring of renetted whole milk during cheese manufacturing. Food Res Int 41:745-50.

Nassar G, Nongaillard B, Noel Y, 2001. Monitoring of milk gelation using a lowfrequency ultrasonic technique. J Food Eng 48:351-9.

Nassar G, Nongaillard B, Noel Y, 2004. Study by ultrasound of the impact of technological parameters changes in the milk gelation process. J Food Eng 63:229-36.

Povey MJW, McClements DJ, 1988. Ultrasonics in food engineering. Part I: introduction and experimental methods. J Food Eng 8:217-45.

Tanter M, Bercoff J, Athanasiou A, Deffieux T, Gennisson JL, Montaldo G, Muller M, Tardivon A, Fink M, 2008. Quantitative assessment of breast lesion viscoelasticity: initial clinical results using supersonic shear imaging. Ultrasound Med Biol 34:1373-86.

Wang Q, Bulca S, Kulozik U, 2007. A comparison of low-intensity ultrasound and oscillating rheology to assess the renneting properties of solutions after UHT heat pretreatment. Int Dairy J 17:50-8. 